Article

\title{
Fungus-Mediated Green Synthesis of Silver Nanoparticles Using Aspergillus terreus
}

\author{
Guangquan $\mathrm{Li}^{1}$, Dan He ${ }^{1}$, Yongqing Qian ${ }^{1}$, Buyuan Guan ${ }^{2}$, Song Gao ${ }^{1}$, Yan Cui ${ }^{1}$, \\ Koji Yokoyama ${ }^{3}$ and Li Wang ${ }^{1, *}$
}

1 Department of Pathogenobiology, Norman Bethune College of Medicine, Jilin University Mycology Research Center, Jilin University, Changchun 130021, China; E-Mails: guangquanli83@gmail.com (G.L.); hedan@jlu.edu.cn (D.H.); qianyq09@mails.jlu.edu.cn(Y.Q.); songgao850118@gmail.com (S.G.); cuiyan923@hotmail.com (Y.C.)

2 State Key Laboratory of Inorganic Synthesis and Preparative Chemistry, College of Chemistry, Jilin University, Changchun 130012, China; E-Mail: guanbuyuan@gmail.com

3 Medical Mycology Research Center, Chiba University, Chiba 260-8673, Japan; E-Mail: yoko@faculty.chiba-u.jp

* Author to whom correspondence should be addressed; E-Mail: wli99@jlu.edu.cn; Tel.: +86-431-85619486; Fax: +86-431-85619402.

Received: 8 November 2011; in revised form: 23 November 2011 / Accepted: 15 December 2011 / Published: 29 December 2011

\begin{abstract}
The biosynthesis of nanoparticles has received increasing attention due to the growing need to develop safe, cost-effective and environmentally friendly technologies for nano-materials synthesis. In this report, silver nanoparticles (AgNPs) were synthesized using a reduction of aqueous $\mathrm{Ag}^{+}$ion with the culture supernatants of Aspergillus terreus. The reaction occurred at ambient temperature and in a few hours. The bioreduction of AgNPs was monitored by ultraviolet-visible spectroscopy, and the AgNPs obtained were characterized by transmission electron microscopy and X-ray diffraction. The synthesized AgNPs were polydispersed spherical particles ranging in size from 1 to $20 \mathrm{~nm}$ and stabilized in the solution. Reduced nicotinamide adenine dinucleotide (NADH) was found to be an important reducing agent for the biosynthesis, and the formation of AgNPs might be an enzyme-mediated extracellular reaction process. Furthermore, the antimicrobial potential of AgNPs was systematically evaluated. The synthesized AgNPs could efficiently inhibit various pathogenic organisms, including bacteria and fungi. The current research opens a new avenue for the green synthesis of nano-materials.
\end{abstract}


Keywords: silver nanoparticles; Aspergillus terreus; biosynthesis; NADH

\section{Introduction}

Nanoparticles (NP) are usually clusters of atoms in the size range of 1-100 nm. It is understood that the properties of a metal NP are determined by its size, shape, composition, crystallinity, and structure [1]. As an important metal, silver nanoparticles (AgNPs) have a number of applications, from electronics [2] and catalysis [3] to infection prevention [4] and medical diagnosis [5]. For example, AgNPs could be used as substrates for Surface Enhanced Raman Scattering (SERS) to probe single molecules [6], and also useful catalysts for the oxidation of methanol to formaldehyde [7]. AgNPs has been known as excellent antimicrobial and anti-inflammatory agents, and thus were used to improve wound healing [8]. To date, a number of physical and chemical strategies were employed for the synthesis of AgNPs [9,10]. However, concern has been raised on the toxicity of chemical agents used in AgNPs synthesis. Thus, it is essential to develop a green approach for AgNPs production without using hazardous substances to the human health and environment.

Compared with the traditional synthetic methods, biological systems provide a novel idea for the production of nano-materials [11]. Up to now, several microorganisms from bacteria to fungi have been reported to synthesize inorganic materials either intra- or extracellularly, and thus to be potentially utilized as eco-friendly nanofactories [12,13]. Pseudomonas stutzeri AG259, isolated from silver mines, has been shown to produce silver nanoparticles [14], and the bioreduction of Ag was also reported in Bacillus licheniformis. Recently a further advancement in the biological synthesis approach was shown by demonstrating that the shape of $\mathrm{Ag}$ nanoparticles could be tuned from nanospheres to nanoprisms by controlling the growth kinetics of a silver resistant bacteria Morganella psychrotolerans [15]. Moreover, the same research group also demonstrated that all the members of the genus Morganella were capable of synthesizing extracellular Ag nanoparticles, which was correlated to silver resistance machinery operating in these organisms [16] Compared with bacteria, fungi have been known to secrete much higher amounts of bioactive substances, which made fungi more suitable for large-scale production [17]. In addition, the extracellular biosynthesis using fungi could also make downstream processing much easier than bacteria [13]. An interesting example of the biosynthesis using fungi was that the cell-associated biosynthesis of silver using Fusarium oxysporum was demonstrated by Ahmad et al., and the particles were overall quasi-spherical with size range between 5 and $15 \mathrm{~nm}$ [18]. There also have been several reports on the biosynthesis of AgNPs using fungi, including Fusarium acuminatum [19] and Penicillium fellutanum [20]. Despite these impressive results, the origins of fungi having the ability for AgNPs synthesis were still limited, and the detailed mechanism was still not well elucidated. Previous reports have shown that a large number of active substances secreted by fungi played important roles as reducing agents and capping agents in the reaction [21]. Therefore, it was of great significance to explore novel fungi strain for synthesizing AgNPs based on the biodiversity. More importantly, it could also facilitate the deeper understanding of molecular mechanism for AgNPs biosynthesis. 
Herein, we investigated the biosynthesis of AgNPs using Aspergillus terreus and its underlying mechanism. The properties of obtained AgNPs were characterized by ultraviolet-visible spectroscopy, transmission electron microscopy (TEM) and X-ray diffraction (XRD) techniques. Furthermore, the key factors controlling the reaction and the antimicrobial activity of AgNPs synthesized were evaluated. This work provided a potential for the production of AgNPs without the involvement of toxic chemicals and radiation.

\section{Results and Discussion}

\subsection{Synthesis and Characterization of AgNPs Using Aspergillus terreus}

In this study, AgNPs were synthesized using a reduction of aqueous $\mathrm{Ag}^{+}$with the culture supernatants of Aspergillus terreus at room temperature.

It was generally recognized that AgNPs produced brown solution in water, due to the surface plasmon resonances (SPR) effect and reduction of $\mathrm{AgNO}_{3}$ [22]. After the addition of $\mathrm{AgNO}_{3}$ solution, the crude cell filtrate of $A$. terreus changed from light yellow to brown in a few hours, while no color change was observed in the culture supernatant without $\mathrm{AgNO}_{3}$ (Figure 1). Thus, color change of the solution clearly indicated the formation of AgNPs. The color intensity of the cell filtrate with $\mathrm{AgNO}_{3}$ was sustained even after $24 \mathrm{~h}$ incubation, which indicated that the particles were well dispersed in the solution, and there was no obvious aggregation.

Figure 1. The crude cell filtrate of Aspergillus terreus mixed without $\mathrm{AgNO}_{3}(\mathbf{A})$ and with $\mathrm{AgNO}_{3}$ (B) after $24 \mathrm{~h}$.

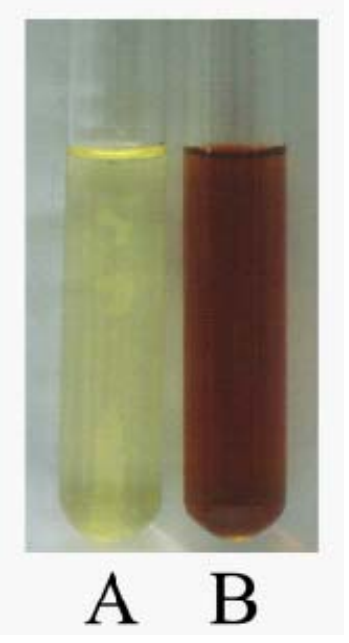

All these reactions were monitored by ultraviolet-visible spectroscopy of the colloidal AgNPs solutions. The ultraviolet-visible spectra of the cell filtrate with $\mathrm{AgNO}_{3}$ showed a strong broad peak at $440 \mathrm{~nm}$ (SPR band), which indicated the presence of AgNPs (Figure 2). These results were consistent with the reports of Naik et al. and Verma et al. [23,24]. The intensity of the SPR band steadily increased from $6 \mathrm{~h}$ to $24 \mathrm{~h}$ as a function of time of reaction. It was also observed that the AgNPs formed were quite stable in the supernatant of A. terreus. 
Figure 2. The UV-Vis spectra recorded for the reaction of fungal cell filtrate with $\mathrm{AgNO}_{3}$ solution.

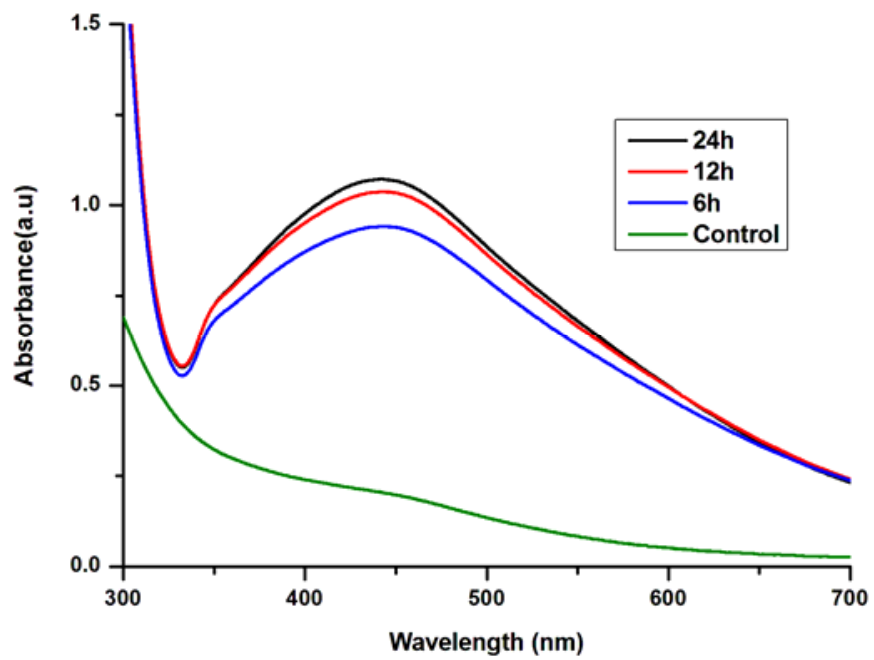

The application of AgNPs was highly dependent on the chemical composition, shape, size, and monodispersity of particles [25]. To broaden the application scope, the AgNPs obtained were systematically characterized using TEM and XRD analysis. Through the TEM analysis, the particles were spherical and polydisperse with an average size of $4.3 \mathrm{~nm}(1-20 \mathrm{~nm})$, and the majority of the particles were less than $10 \mathrm{~nm}$ (Figure 3). For the crystalline nature of the AgNPs, intense XRD peaks were observed corresponding to the (111), (200), (220), (311) planes at $2 \theta$ angles of $38.28^{\circ}, 44.38^{\circ}$, $64.54^{\circ}$, and $77.64^{\circ}$, respectively (Figure 4). This was in good agreement with the unit cell of the face centered cubic (fcc) structure (JCPDS File No. 04-0783) with a lattice parameter of a $=4.077 \AA$. Some intense diffraction peaks at $2 \theta$ angles of $32.05^{\circ}, 46.05^{\circ}, 54.6^{\circ}$ and $57.3^{\circ}$, might be related to $\mathrm{AgCl}$ which was owing to the chloride ions involved during preparation of the cell filtrate. Because of the biomass residue, other crystallographic impurities were also observed in the XRD profile. The size of AgNPs according to the XRD was about $5.2 \mathrm{~nm}$. This result was consistent with the TEM study.

Figure 3. (A) Representative images of AgNPs synthesized by the reduction of $\mathrm{AgNO}_{3}$ solution with the crude cell filtrate from Aspergillus terreus; (B) Size distribution of the AgNPs from TEM analysis.
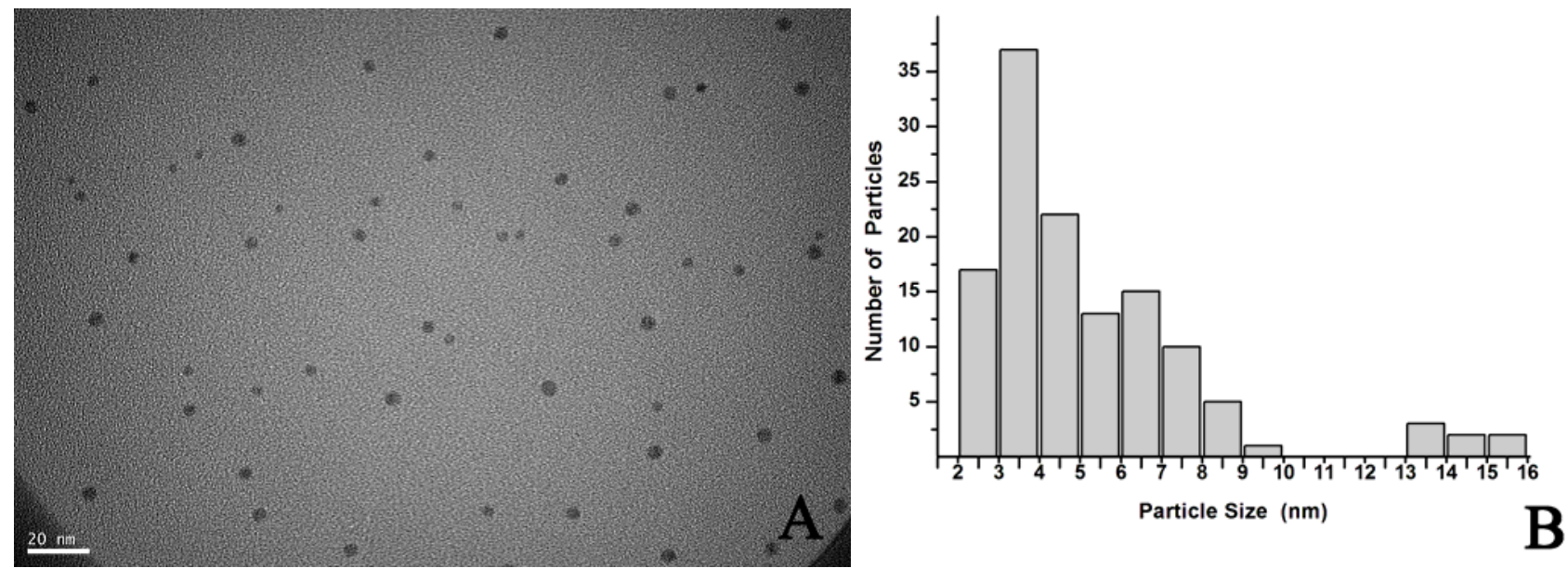
Figure 4. Representative X-ray diffraction patterns of AgNPs synthesized by Aspergillus terreus (a.u. = arbitrary units).

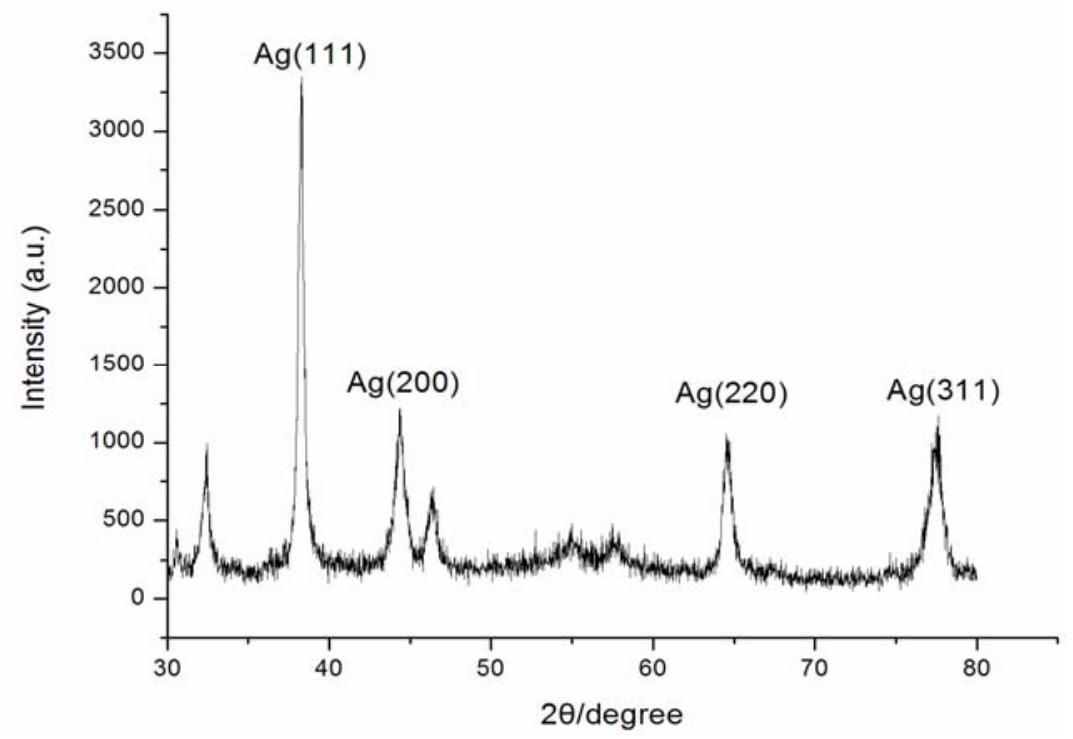

\subsection{Key Factors Governing the Biosynthesis of AgNPs}

In the present study, the key factors in the supernatant of $A$. terreus governing the formation of AgNPs were investigated. The $\mathrm{AgNO}_{3}$ solution was added to the dialyzed cell filtrate under the same conditions for the synthesis of AgNPs. However, the color of reaction mixture did not change over $24 \mathrm{~h}$. Meanwhile, the absence of SPR at $440 \mathrm{~nm}$ for the reaction mixture of dialyzed cell filtrate (Figure 5C) indicated that the reaction was highly dependent on an active substance with a low molecular weight ( $<7000 \mathrm{Da})$. Interestingly, when NADH was added to the dialyzed cell filtrate, the reaction was recovered in a few minutes, and the band at $440 \mathrm{~nm}$ was almost as strong as that in the crude cell filtrate (Figure 5A). These results indicated that NADH might be a key factor for the synthesis of AgNPs by A. terreus. As a negative control, when NADH alone was added to the $\mathrm{AgNO}_{3}$ solution, no band was observed at $440 \mathrm{~nm}$ (Figure 5B), which confirmed that NADH alone was not sufficient for the reaction to occur, and other active substances with higher molecular weight might also play important roles in the reduction of silver ions.

In organism, NADH is a widespread reduced coenzyme involved in redox reaction, and can be used as a reducing agent by many enzymes in vivo [26,27]. Thus, we interfered that a NADH-dependent reductase released by $A$. terreus might account for the synthesis of AgNPs. In the process, NADH acted as an electron carrier, and the silver ions obtained electrons from NADH via the NADH-dependent reductase, and then were reduced to $\mathrm{Ag}$ (Figure 6). Similarly, there were other reports that NADH-dependent reductases were believed to be involved in the biosynthesis of AgNPs in F. oxysporum and P. fellutanum [20,28,29]. These findings might be of great significance to the development of a continuous biological production of nanoparticles at a large scale through the addition of NADH or couple with the regenerating system of co-enzymes. 
Figure 5. The UV-Vis spectra recorded for the reaction of dialyzed fungal cell filtrate with $\mathrm{AgNO}_{3}$ solution. Curve A corresponds to the dialyzed cell filtrate with $\mathrm{NADH}$ and $\mathrm{AgNO}_{3}$ solution; Curve $\mathrm{B}$ corresponds to $\mathrm{NADH}$ alone with $\mathrm{AgNO}_{3}$ solution; Curve $\mathrm{C}$ corresponds to the dialyzed cell filtrate with $\mathrm{AgNO}_{3}$ solution.

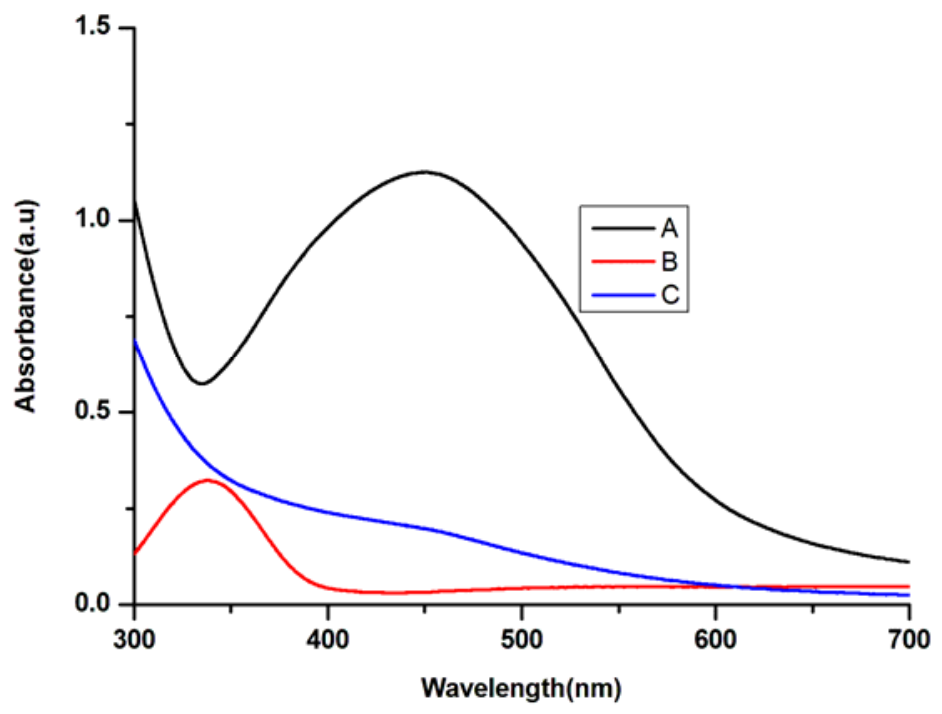

Figure 6. Schematic representation of the biosynthesis of AgNPs related to NADH, NADH-dependent reductase was also essential for the reaction.

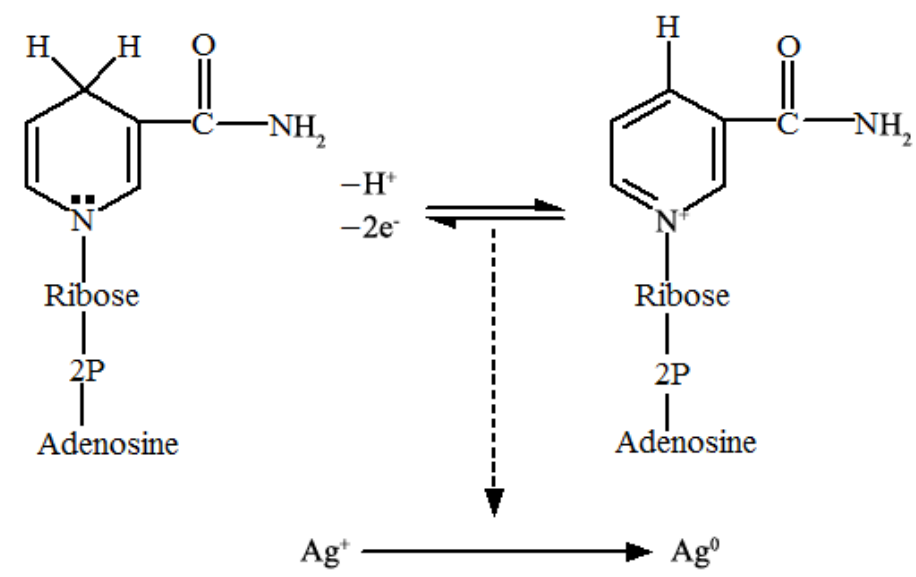

Representative TEM images of AgNPs film which was derived from dialyzed cell filtrate with the addition of NADH were shown in Figure 7. The AgNPs were spherical or nearly spherical, which was same as the ones synthesized by crude cell filtrate. Nevertheless, the size of nanospheres was about $21.3 \mathrm{~nm}$ on average and in the range of $10-30 \mathrm{~nm}$, a little larger than the ones synthesized by crude cell filtrate. This size difference might be caused by different concentrations of NADH in these two reaction mixtures, and the presence or absence of other small molecules (might as stabilizers). Therefore, the concentration of reducing agent and the presence of stabilizer were crucial to the size and shape control of nanoparticles in such a biosynthesis system. 
Figure 7. (A) Representative images of AgNPs synthesized by the reduction of $\mathrm{AgNO}_{3}$ solution with the dialyzed cell filtrate and NADH; (B) Size distribution of the AgNPs from TEM analysis.
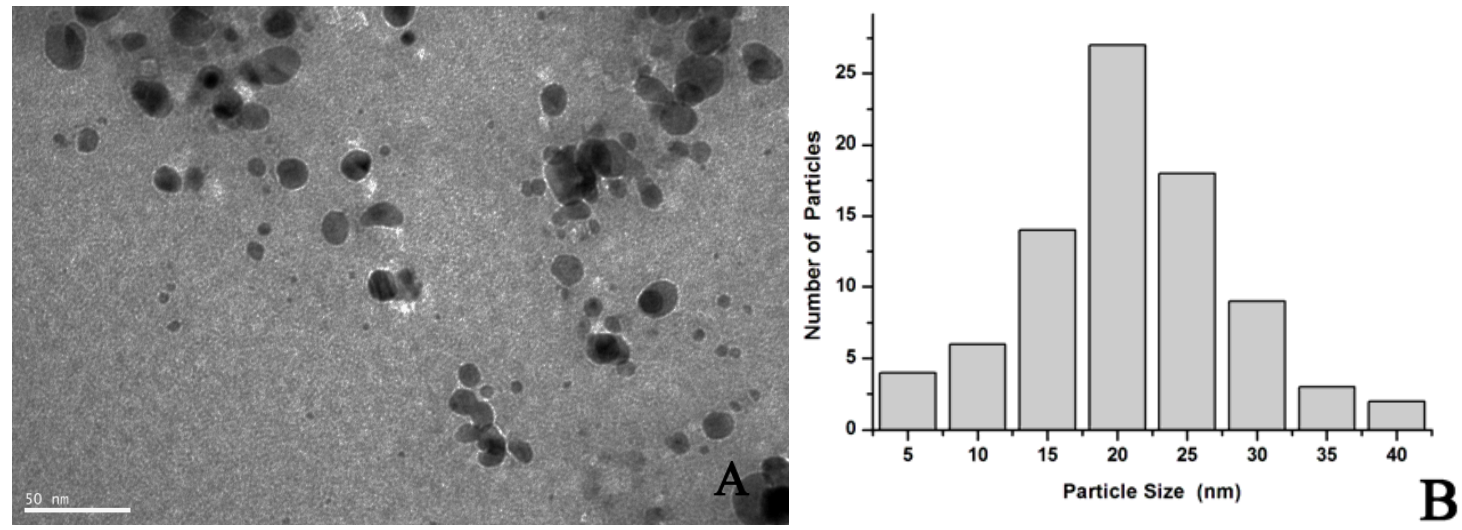

\subsection{Antimicrobial Activity Analysis of AgNPs}

The antimicrobial activity of AgNPs against various pathogenic organisms including bacteria and fungi was investigated. Compared with the control, the diameters of inhibition zones increased for all the test pathogens (Table 1). The AgNPs produced could inhibit three different typical pathogenic bacteria, including Staphylococcus aureu, Pseudomonas aeruginosa and Escherichia coli, as previously described [23,30]. Thus, AgNPs could be considered as excellent broad-spectrum antibacterial agents. More importantly, the AgNPs produced by A. terreus exhibited potent antifungal activity against Candida species, which were the most important pathogenic fungi. Additionally, the AgNPs showed good inhibition activity towards two kinds of filamentous fungus, which were naturally resistant to the common antifungal agent Fluconazole [31]. Since the biosynthesized AgNPs showed considerable antifungal activity, they could be potential to be widely used in clinical applications.

Table 1. Size of the inhibition zone for AgNPs synthesized by Aspergillus terreus against the tested microorganisms.

\begin{tabular}{lcc}
\hline \multirow{2}{*}{ Tested Pathogenic Organisms } & \multicolumn{2}{c}{ Mean Size of Inhibition Zone (mm) } \\
\cline { 2 - 3 } Candida albicans (ATCC 90028) & Control & Test \\
Candida krusei (ATCC 6258) & 9 & $16 \pm 1$ \\
Candida parapsilosis (ATCC 22019) & 10 & $14 \pm 2$ \\
Candida tropicalis (JLCC 30394) & 9 & $13 \pm 1$ \\
Aspergillus flavus (IFM 55648) & 10 & $14 \pm 1$ \\
Aspergillus fumigates (IFM 40808) & 9 & $13 \pm 2$ \\
Staphylococcus aureus (ATCC 25923) & 9 & $14 \pm 2$ \\
Pseudomonas aeruginosa (ATCC 27853) & 9 & $16 \pm 1$ \\
Escherichia coli (ATCC 25922) & 9 & $12 \pm 1$ \\
\hline
\end{tabular}

ATCC: American Type Culture Collection, USA; IFM: Institute for Food Microbiology (at present the Medical Mycology Research Center, Chiba University), Japan; JLCC: Culture Collection of Jilin University, Mycology Research Center, China; Control: $\mathrm{AgNO}_{3}$; Test: AgNPs. 


\section{Experimental Section}

\subsection{Materials}

A. terreus (BC0603) was isolated from soil, and maintained on potato dextrose agar (PDA) medium at $28{ }^{\circ} \mathrm{C}$. The isolated fungus was identified using morphological characteristics and mitochondrial cytochrome $b$ gene analysis [32]. Three kinds of bacteria were tested for their susceptibility for AgNPs: S. aureu, E. coli and P. aeruginosa. Six kinds of fungi were all tested for its antifungal effect: C. albicans, C. parapsilosis, C. krusei, C. tropicalis, A. fumigatus and A. flavus. A. terreus and all strains used in the study were stored in Jilin University Mycology Research Center (JUMRC).

Two medium (potato dextrose agar and potato dextrose broth) were purchased from BD (Becton, Dickinson and company Co., Sparks, MD, USA). The chemical silver nitrate $\left(\mathrm{AgNO}_{3}\right)$ and $\mathrm{NADH}$ were purchased from Sigma-Aldrich (St. Louis, MO, USA), and used as received.

\subsection{Biomass Preparation}

To prepare biomass for biosynthesis, A. terreus was grown in potato dextrose broth liquid medium (PDB). The flasks were inoculated with spores and incubated at $28{ }^{\circ} \mathrm{C}$ on a rotary shaker $(120 \mathrm{rpm})$ for $96 \mathrm{~h}$. The biomass was harvested by filtration through filter paper (Whatman filter paper No. 1), and then washed with distilled water to remove any components of the medium. $25 \mathrm{~g}$ biomass (wet weight) was placed in individual flasks containing $100 \mathrm{~mL}$ Milli-Q water. The flasks were incubated under the conditions described above for $24 \mathrm{~h}$. The biomass was filtered, and the crude cell filtrate was collected for subsequent experiment.

\subsection{Biosynthesis of AgNPs}

AgNPs were synthesized using $50 \mathrm{~mL}$ cell filtrate mixed with $10 \mathrm{~mL} \mathrm{AgNO}_{3}$ solution $(10 \mathrm{mmol} / \mathrm{L})$ in a $250 \mathrm{~mL}$ Erlenmeyer flask incubated at $28{ }^{\circ} \mathrm{C}$ in dark for $24 \mathrm{~h}$. A flask with no addition of silver ion was used as control. AgNPs were concentrated by centrifugation of the reaction mixture at $10,000 \mathrm{rpm}$ for $10 \mathrm{~min}$ twice, and then were collected for further characterization.

\subsection{Characterization of AgNPs}

The bioreduction of $\mathrm{Ag}^{+}$in aqueous solution was monitored using an ultraviolet-visible spectrophotometer (Shimadzu UV-2550) from 240 to $750 \mathrm{~nm}$, at a resolution of $1 \mathrm{~nm}$.

The dried reaction mixture embedded with AgNPs was used for XRD analysis. XRD patterns were recorded on RINT2000 vertical goniometer operated at a voltage of $50 \mathrm{kV}$ and current of $200 \mathrm{~mA}$ with $\mathrm{Cu} \mathrm{K} \alpha$ radiation $(\lambda=1.5405 \AA)$, and the diffracted intensities were recorded from $30^{\circ}$ to $80^{\circ} 2 \theta$ angles.

For TEM analysis, a drop of aqueous solution containing AgNPs were placed on the carbon coated copper grids and dried by allowing water to evaporate at room temperature. Micrographs were obtained using a Tecnai F20 S-Twin (USA) operating at $200 \mathrm{kV}$. The size of AgNPs were estimated from the Debye-Scherrer Eq by determine the width of the (111) Bragg reflection [2,33], and size distribution of the resulting nanoparticles was also estimated on the basis of TEM micrographs. 


\subsection{Investigation of the Key Factors of Reaction}

To investigate the key factors of reaction, some of crude cell filtrate was dialyzed (molecular weight cut-off $=7 \mathrm{kDa}$ ) against distilled water for $48 \mathrm{~h}$ at $4{ }^{\circ} \mathrm{C}$ to remove small molecular weight compounds. $200 \mu \mathrm{L} \mathrm{NADH}(20 \mathrm{mmol} / \mathrm{L})$ was then added to the dialyzed cell filtrate. Afterwards, $\mathrm{AgNO}_{3}$ solution $(10 \mathrm{mmol} / \mathrm{L})$ was added under the same conditions as above. Control reactions were performed without the addition of cell filtrate or NADH.

\subsection{The Antimicrobial Activity Analysis of AgNPs}

The antimicrobial activity of AgNPs synthesized from A. terreus against P. aeruginosa, S. aureus, E. coli, C. albicans, C. krusei, C. glabrata, C. tropicalis, A. fumigatus and A. flavus was investigated using a disk diffusion assay. The disk diffusion assay was carried out using the Oxford cup method [34]. Each strain was swabbed uniformly onto individual plates, and a concentrated solution of AgNPs was poured into each cup $\left(20 \mu \mathrm{g}\right.$ per cup) on all the plates. After incubation at $37{ }^{\circ} \mathrm{C}$ or $28{ }^{\circ} \mathrm{C}$ for $24 \mathrm{~h}$, the diameter of inhibition zone was measured using caliper. $\mathrm{AgNO}_{3}(10 \mathrm{mmol} / \mathrm{L})$ was used individually as the negative control. The assays were performed in triplicate.

\section{Conclusions}

In this study, AgNPs were synthesized extracellularly by A. terreus at room temperature. The AgNPs were quite stable without using any toxic chemicals as capping agents. The spherical AgNPs ranged in size from 1 to $20 \mathrm{~nm}$, and showed promising broad-spectrum antimicrobial activity. NADH and NADH-dependent reductase were probably the key factors for the biosynthesis of AgNPs. The ability to synthesize AgNPs as potential anti-microbial agents using A. terreus is highly promising for the green, sustainable production of nano-metals, and also enhances its widespread application as an important strategy.

\section{Acknowledgments}

We are grateful to Qisheng Huo for providing facilities from state key laboratory of inorganic synthesis and preparative chemistry, college of chemistry, Jilin University. This research was supported by the grants from National Natural Science Foundation of major international cooperation projects of China (No. 30910103903) and Graduate Innovation Fund of Jilin University.

\section{References}

1. Sun, Y.G.; Xia, Y.N. Shape-controlled synthesis of gold and silver nanoparticles. Science 2002, 298, 2176-2179.

2. Gratzel, M. Photoelectrochemical cells. Nature 2001, 414, 338-344.

3. Shiraishi, Y.; Toshima, N. Colloidal silver catalysts for oxidation of ethylene. J. Mol. Catal. A 1999, 141, 187-192.

4. White, R.J.; Budarin, V.L.; Moir, J.W.B.; Clark, J.H. A sweet killer: Mesoporous polysaccharide confined silver nanoparticles for antibacterial applications. Int. J. Mol. Sci. 2011, 12, 5782-5796. 
5. Groneberg, D.A.; Giersig, M.; Welte, T.; Pison, U. Nanoparticle-based diagnosis and therapy. Curr. Drug Targets 2006, 7, 643-648.

6. Tao, A.; Kim, F.; Hess, C.; Goldberger, J.; He, R.R.; Sun, Y.G.; Xia, Y.N.; Yang, P.D. Langmuir-blodgett silver nanowire monolayers for molecular sensing using surface-enhanced raman spectroscopy. Nano Lett. 2003, 3, 1229-1233.

7. Waterhouse, G.I.N.; Bowmaker, G.A.; Metson, J.B. Oxygen chemisorption on an electrolytic silver catalyst: A combined TPD and raman spectroscopic study. Appl. Surf. Sci. 2003, 214, 36-51.

8. Elliott, C. The effects of silver dressings on chronic and burns wound healing. Br. J. Nurs. 2010, 19, S32-S36.

9. Darroudi, M.; Ahmad, M.B.; Zak, A.K.; Zamiri, R.; Hakimi, M. Fabrication and characterization of gelatin stabilized silver nanoparticles under UV-light. Int. J. Mol. Sci. 2011, 12, 6346-6356.

10. Kilin, D.S.; Prezhdo, O.V.; Xia, Y.N. Shape-controlled synthesis of silver nanoparticles: Ab initio study of preferential surface coordination with citric acid. Chem. Phys. Lett. 2008, 458, 113-116.

11. Bansal, V.; Ramanathan, R.; Bhargava, S.K. Fungus-mediated biological approaches towards "green" synthesis of oxide nanomaterials. Aust. J. Chem. 2011, 64, 279-293.

12. Shankar, S.S.; Rai, A.; Ankamwar, B.; Singh, A.; Ahmad, A.; Sastry, M. Biological synthesis of triangular gold nanoprisms. Nat. Mater. 2004, 3, 482-488.

13. Mohanpuria, P.; Rana, N.K.; Yadav, S.K. Biosynthesis of nanoparticles: Technological concepts and future applications. J. Nanopart. Res. 2008, 10, 507-517.

14. Klaus, T.; Joerger, R.; Olsson, E.; Granqvist, C.G. Silver-based crystalline nanoparticles, microbially fabricated. Proc. Natl. Acad. Sci. USA 1999, 96, 13611-13614.

15. Ramanathan, R.; O’Mullane, A.P.; Parikh, R.Y.; Smooker, P.M.; Bhargava, S.K.; Bansal, V. Bacterial kinetics-controlled shape-directed biosynthesis of silver nanoplates using morganella psychrotolerans. Langmuir 2011, 27, 714-719.

16. Parikh, R.Y.; Ramanathan, R.; Coloe, P.J.; Bhargava, S.K.; Patole, M.S.; Shouche, Y.S.; Bansal, V. Genus-wide physicochemical evidence of extracellular crystalline silver nanoparticles biosynthesis by Morganella spp. PLoS One 2011, 6, e21401.

17. Narayanan, K.B.; Sakthivel, N. Biological synthesis of metal nanoparticles by microbes. Adv. Colloid Interface Sci. 2010, 156, 1-13.

18. Ahmad, A.; Mukherjee, P.; Senapati, S.; Mandal, D.; Khan, M.I.; Kumar, R.; Sastry, M. Extracellular biosynthesis of silver nanoparticles using the fungus Fusarium oxysporum. Colloid Surface B 2003, 28, 313-318.

19. Ingle, A.; Gade, A.; Pierrat, S.; Sonnichsen, C.; Rai, M. Mycosynthesis of silver nanoparticles using the fungus Fusarium acuminatum and its activity against some human pathogenic bacteria. Curr. Nanosci. 2008, 4, 141-144.

20. Kathiresan, K.; Manivannan, S.; Nabeel, M.A.; Dhivya, B. Studies on silver nanoparticles synthesized by a marine fungus, Penicillium fellutanum isolated from coastal mangrove sediment. Colloid Surface B 2009, 71, 133-137.

21. Bharde, A.; Rautaray, D.; Bansal, V.; Ahmad, A.; Sarkar, I.; Yusuf, S.M.; Sanyal, M.; Sastry, M. Extracellular biosynthesis of magnetite using fungi. Small 2006, 2, 135-141. 
22. Wiley, B.J.; Im S.H.; Li, Z.Y.; McLellan, J.; Siekkinen, A.; Xia, Y. Maneuvering the surface plasmon resonance of silver nanostructures through shape-controlled synthesis. J. Phys. Chem. B 2006, 110, 15666-15675.

23. Verma, V.C.; Kharwar, R.N.; Gange, A.C. Biosynthesis of antimicrobial silver nanoparticles by the endophytic fungus Aspergillus clavatus. Nanomedicine (Lond) 2010, 5, 33-40.

24. Huang, J.L.; Li, Q.B.; Sun, D.H.; Lu, Y.H.; Su, Y.B.; Yang, X.; Wang, H.X.; Wang, Y.P.; Shao, W.Y.; He, N.; et al. Biosynthesis of silver and gold nanoparticles by novel sundried Cinnamomum camphora leaf. Nanotechnology 2007, 18, doi:10.1088/0957-4484/18/10/105104.

25. Bansal, V.; Li, V.; O’Mullane, A.P.; Bhargava, S.K. Shape dependent electrocatalytic behaviour of silver nanoparticles. CrystEngComm 2010, 12, 4280-4286.

26. Dudev, T.; Lim, C. Factors controlling the mechanism of NAD ${ }^{+}$non-redox reactions. J. Am. Chem. Soc. 2010, 132, 16533-16543.

27. Lin, H. Nicotinamide adenine dinucleotide: Beyond a redox coenzyme. Org. Biomol. Chem. 2007, 5, 2541-2554.

28. Ahmad, A.; Mukherjee, P.; Mandal, D.; Senapati, S.; Khan, M.I.; Kumar, R.; Sastry, M. Enzyme mediated extracellular synthesis of $\mathrm{CdS}$ nanoparticles by the fungus, Fusarium oxysporum. J. Am. Chem. Soc. 2002, 124, 12108-12109.

29. Govender, Y.; Riddin, T.L.; Gericke, M.; Whiteley, C.G. On the enzymatic formation of platinum nanoparticles. J. Nanopart. Res. 2010, 12, 261-271.

30. Khan, S.S.; Mukherjee, A.; Chandrasekaran, N. Studies on interaction of colloidal silver nanoparticles (SNPs) with five different bacterial species. Colloid Surface B 2011, 87, 129-138.

31. Espinel-Ingroff, A.; Warnock, D.W.; Vazquez, J.A.; Arthington-Skaggs, B.A. In vitro antifungal susceptibility methods and clinical implications of antifungal resistance. Med. Mycol. 2000, 38, 293-304.

32. Wang, L.; Yokoyama, K.; Miyaji, M.; Nishimura, K. The identification and phylogenetic relationship of pathogenic species of Aspergillus based on the mitochondrial cytochrome $b$ gene. Med. Mycol. 1998, 36, 153-164.

33. Borchert, H.; Shevehenko, E.V.; Robert, A.; Mekis, I.; Kornowski, A.; Grubel, G.; Weller, H. Determination of nanocrystal sizes: A comparison of TEM, SAXS, and XRD studies of highly monodisperse $\mathrm{CoPt}_{3}$ particles. Langmuir 2005, 21, 1931-1936.

34. Wang, Y.; Lu, Z.; Wu, H.; Lv, F. Study on the antibiotic activity of microcapsule curcumin against foodborne pathogens. Int. J. Food. Microbiol. 2009, 136, 71-74.

(C) 2012 by the authors; licensee MDPI, Basel, Switzerland. This article is an open access article distributed under the terms and conditions of the Creative Commons Attribution license (http://creativecommons.org/licenses/by/3.0/). 